\title{
Cumulative Review of Thrombotic Microangiopathy, Thrombotic Thrombocytopenic Purpura, and Hemolytic Uremic Syndrome Reports with Subcutaneous Interferon $\beta$-1a
}

\author{
Ali-Frédéric Ben-Amor · Anton Trochanov • Tanya Z. Fischer
}

To view enhanced content go to www.advancesintherapy.com

Received: March 16, 2015 / Published online: May 20, 2015

(C) The Author(s) 2015. This article is published with open access at Springerlink.com

\section{ABSTRACT}

Introduction: Rare cases of thrombotic microangiopathy (TMA), manifested as thrombotic thrombocytopenic purpura (TTP) or hemolytic uremic syndrome (HUS), have been reported with interferon $\beta$ products. We performed a cumulative review of TMA cases recorded in a Global Safety Database for patients with multiple sclerosis who received subcutaneous interferon $\beta$-1a treatment.

Methods: Search criteria were: all reported cases, serious and non-serious, from all sources (including non-health care professionals and

Parts of these data were presented as posters at the Middle East North Africa Committee of Treatment and Research in Multiple Sclerosis (MENACTRIMS) 2014 Congress, Dubai, UAE, and the American Academy of Neurology (AAN) 2015 Annual Meeting, Washington, USA.

Electronic supplementary material The online version of this article (doi:10.1007/s12325-015-0212-6) contains supplementary material, which is available to authorized users.

A.-F. Ben-Amor $(\square)$

Merck Serono, Ares Trading SA, Aubonne,

Switzerland

e-mail: ali-frederic.ben-amor@merckgroup.com

A. Trochanov · T. Z. Fischer

EMD Serono, Inc, Billerica, MA, USA clinical trial reports), regardless of event ranking and causality assessment by reporter or company. Data lock was May 3, 2014, with additional analysis of cases reported between August 1, 2014-November 30, 2014.

Results: Ninety-one patient cases (76.9\% female) with 105 events were retrieved. Time to onset varied from 2 months to 14 years, and in $31.9 \%$ of patients the event occurred within 2 years of treatment initiation. Seven patients had a fatal outcome (five were secondary to other causes and two reported insufficient information). Forty-four patients recovered, 32 patients had not recovered at the time of the report, and in eight cases outcome was either not reported or unknown. Treatment was discontinued in $84.6 \%$ (77/91) of patients. In $67 \%(61 / 91)$ of patients, the reporter suspected a causal association between treatment and TMA/TTP-HUS. Risk factors and/or confounding factors were present in $45.1 \%$ (41/91) of patients. Early prodromal syndrome or specific patterns were not detected, although $54.9 \%(50 / 91)$ of cases contained insufficient information. Overall reporting rate of TMA/ TTP-HUS was estimated as 7.2 per 100,000 patient-years. Reporting rates for human 
serum album (HSA)-containing and HSA-free formulations were 5.72 and 7.68 per 100,000 patient-years, respectively.

Conclusion: No new signal relating specifically to increased frequency of TMA/TTP-HUS with HSA-free subcutaneous interferon $\beta-1 \mathrm{a}$ was detected and no additional risk mitigation measures are required regarding the different formulations. The benefit-risk balance of subcutaneous interferon $\beta$-1a remains positive, and routine pharmacovigilance monitoring is appropriate.

Funding: Ares Trading SA, Aubonne, Switzerland, a subsidiary of Merck Serono SA.

Keywords: Hemolytic uremic syndrome; Interferon $\beta-1 \mathrm{a}$; Thrombotic microangiopathy; Thrombotic thrombocytopenic purpura

\section{INTRODUCTION}

Interferon (IFN) $\beta$ is a widely prescribed recombinant-protein therapy with a wellestablished, favorable safety profile. However, rare cases of thrombotic microangiopathy (TMA) have been associated with different IFN $\beta$ formulations, including intramuscular IFN $\beta$ $1 \mathrm{a}$, subcutaneous (sc) IFN $\beta-1 \mathrm{a}$, and sc IFN $\beta-1 \mathrm{~b}$ $[1,2]$.

TMA is a pathology expressed as thrombocytopenia, microangiopathic hemolytic anemia, and microvascular occlusion. TMA is common to a number of disorders, including hemolytic uremic syndrome (HUS), thrombotic thrombocytopenic purpura (TTP), malignant hypertension, and renal transplantation. TTP and HUS have distinct pathophysiologies and diagnoses, but there is considerable clinical overlap [3]. In many case reports and series, diagnostic uncertainty exists as ADAMTS 13 (von Willebrand factor-cleaving protease) levels are generally not available, prompting the use of the composite term TTP-HUS [3]. TTP-HUS is characterized by renal insufficiency, neurologic impairment, and fever [4], and risk factors include older age [5], female gender [5], systemic lupus erythematosus [6], shiga toxin-producing bacteria (e.g., Escherichia coli) [3], and concomitant exposure to other treatments (e.g., chemotherapeutic agents, quinine/thiamine, ticlopidine, ibuprofen) $[7,8]$.

The use of sc IFN $\beta$-1a $\left(\right.$ Rebif $^{\circledR}$, EMD Serono Inc.) is approved for the treatment of patients with relapsing forms of multiple sclerosis (MS) [9]. sc IFN $\beta-1 \mathrm{a}$ has been marketed as a formulation containing human serum albumin (HSA) since February 5, 1998 in Canada, May 4, 1998 in the European Union (EU), and March 7, 2002 in the USA [9]. Since August and September 2007, a HSA-free formulation of sc IFN $\beta$ - $1 \mathrm{a}$ has been available in the $\mathrm{EU}$ and Canada, respectively. The risk of TMA has been described in the summary of product characteristics (SmPC) for sc IFN $\beta$-1a since 2008 [10], and was included in both the EU and US labels. More recently, a cluster of four TMA cases associated with HSA-free sc IFN $\beta-1$ a was reported in South Scotland from 2010 to 2012 [11].

The aim of this cumulative review was to characterize reports of TMA and TTP-HUS for patients with MS who received sc IFN $\beta-1 \mathrm{a}$ (Rebif) from a Global Safety Database, and to determine whether any differences in incidence rates were associated with the introduction of the HSA-free formulation of sc IFN $\beta-1$ a in 2007.

\section{METHODS}

Reported cases of TMA, TTP, and HUS in patients with MS who received sc IFN $\beta-1 \mathrm{a}$ (Rebif) were cumulatively reviewed in the 
Global Safety Database (Adverse Reaction Information System global, ARISg; ArisGlobal, CT, USA) for sc IFN $\beta$-1a (Rebif) treatment, which comprises data for adverse events (AEs) from clinical trials and post-marketing surveillance reports. Search criteria were: all reported cases, serious and non-serious, from all sources (including non-health care professionals and clinical trial reports), regardless of event ranking and causality assessment by reporter or company. The database was searched using a custom Medical Dictionary for Regulatory Activities (MedDRA v17.0; the International Federation of Pharmaceutical Manufacturers \& Associations, VA, USA) search strategy recommended by the Pharmacovigilance Risk Assessment Committee (European Medicines Agency) and developed to capture TMA, TTP, and HUS, using the following MedDRA codes: thrombotic thrombocytopenic purpura, hemolytic uremic syndrome, thrombocytopenic purpura, thrombotic microangiopathy, idiopathic thrombocytopenic purpura, immune thrombocytopenic purpura.

A data lock of May 3, 2014 was used to account for the most recent Periodic Safety Update Report. In addition, a time period of August 1, 2014-November 30, 2014 was used to assess the impact of reporting rates of TMA/TTPHUS following the circulation of a Dear Healthcare Provider (DHCP) letter. At the request of the European Medicines Agency, the DHCP letter detailed the risks of, and need to monitor for, TTP-HUS in patients treated with sc IFN $\beta-1 \mathrm{a}$, and was circulated to healthcare professionals between August 12, 2014 and October 3, 2014 by manufacturers of sc IFN $\beta$ 1a (Bayer, Biogen Idec, Merck Serono, Novartis).

Overall and formulation (HSA-containing and HSA-free) reporting rates for sc IFN $\beta-1 \mathrm{a}$ were calculated using medically and non- medically confirmed patient cases and patientexposure data. Patient-exposure data were derived from an internal sales source and based on the number of sc IFN $\beta$-1a syringes sold over a period of time. The HSA-containing formulation reporting rate was calculated using: total exposure minus HSA-free sc IFN $\beta-1 \mathrm{a}$ exposure. Calculation of formulation reporting rates required assigning all patient case reports to the relevant formulation. As the HSA-free formulation is not available in the USA, all US patient case reports were assigned to the HSAcontaining formulation. In countries where the HSA-free formulation has replaced the HSAcontaining formulation, all events with an onset date $\geq 6$ months after the launch of the new formulation were assumed to be related to the HSA-free formulation.

The analysis in this article is based on previously conducted clinical studies and postmarketing surveillance reports, and does not involve any new studies of human subjects or animals. There was no change to the medical management of patients, and no randomization of participants to different groups.

Statistical analyses are descriptive and further analyses of data were not feasible as the Global Safety Database includes a population of uncertain size.

\section{RESULTS}

\section{Case Presentation: Data Lock May 3, 2014}

In total, 91 serious patient cases were retrieved with 105 serious events of TMA, TTP, and HUS associated with sc IFN $\beta$-1a treatment; $83.8 \%$ $(n=88 / 105)$ of events were medically confirmed and $16.2 \% \quad(n=17 / 105)$ nonmedically confirmed. No patient cases or events were non-serious. Most patients 
$(n=70 / 91 ; 76.9 \%)$ were female and mean age was 44.5 years (range $17-73$ years). Time to onset ranged from 2 months to 14 years with a mean of 4.5 years $(n=91$; unknown $=20)$. In $31.9 \%(n=29 / 91)$ of patient cases, the event occurred within the first 2 years of treatment.

The distribution of patient cases reporting an event according to formulation and year of receipt generally increased over the defined search period (Fig. 1). Patient cases were geographically distributed across five continents and more than half of cases were reported in France and the USA: 33.0\% USA;
20.9\% France; $12.1 \%$ UK; 9.9\% Germany; 7.6\% Canada; $4.4 \%$ Italy; $2.2 \%$ Spain; and $1.1 \%$ each for Austria, Brazil, Finland, Greece, Iran, Japan, Slovenia, Switzerland, Turkey.

Broadly, the search terms TMA, TTP, HUS, and TP retrieved a similar number of patient cases (Fig. 2). Seven of 91 patients reported TMA with either TTP or HUS and 13 cases without TTP or HUS. The non-search preferred terms renal failure $(n=22 / 91 ; 24.2 \%) \quad$ and hypertension $\quad(n=15 / 91 ; 16.5 \%) \quad$ were relatively frequent, while other non-search preferred terms were relatively rare (Table S1

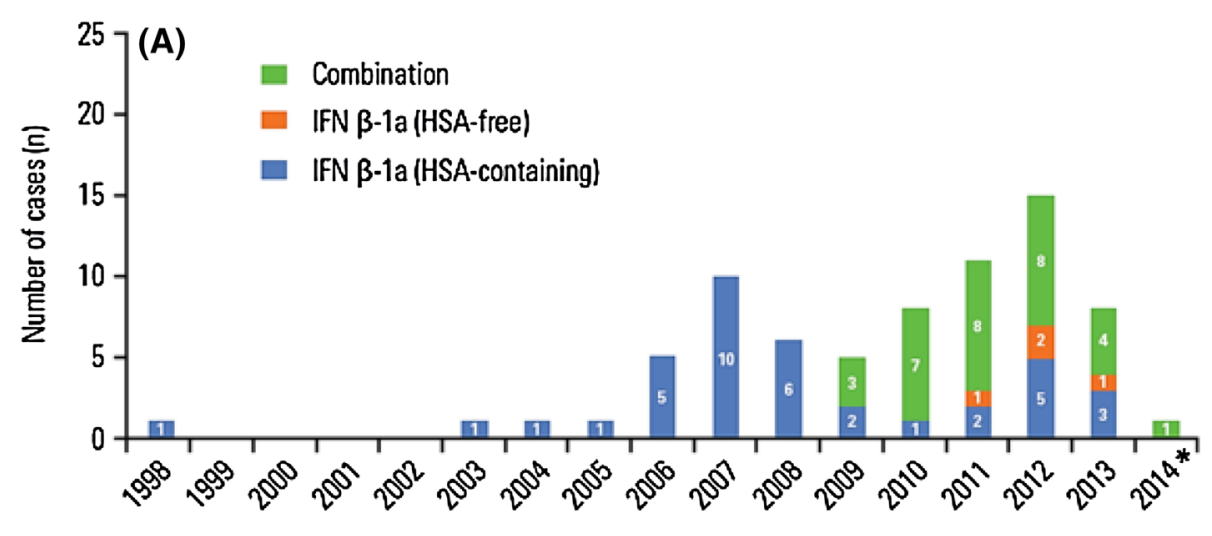

Year of onset

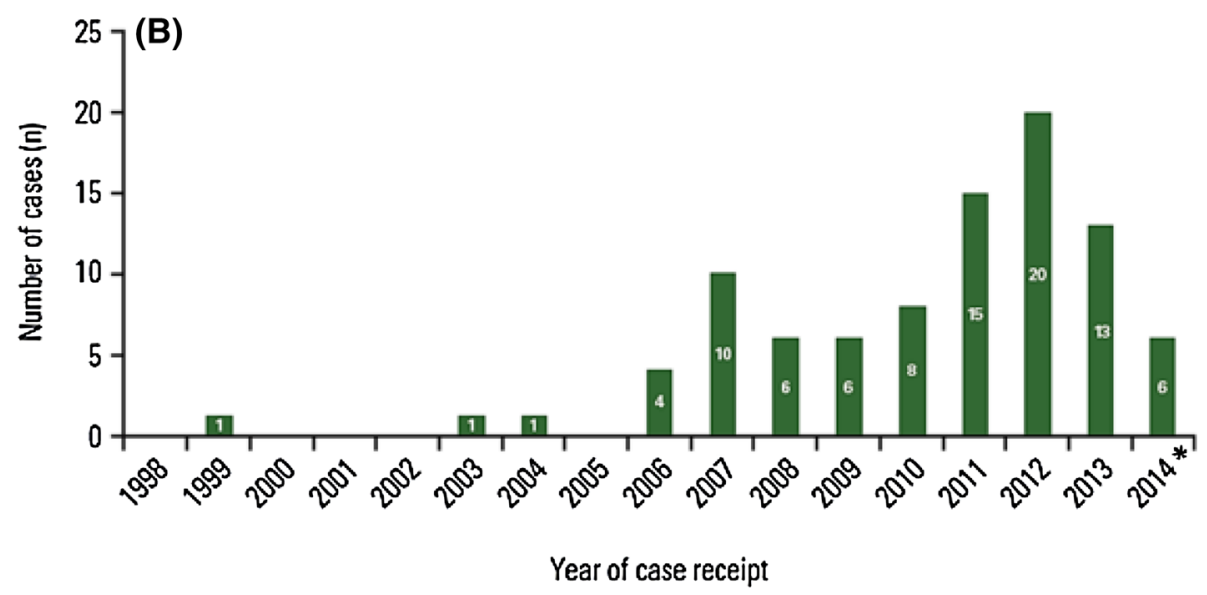

Fig. 1 Distribution of patient cases. a Distribution by year of onset according to the formulations of sc IFN $\beta$-1a (not shown are the 18 cases which were reported with no date of adverse event onset: IFN $\beta$ - 1 a formulation not known, $n=10$; HSA-containing sc IFN $\beta-1 \mathrm{a}, n=7$; HSA-free sc
IFN $\beta$-1a, $n=1$; combination, $n=0)$. $\mathbf{b}$ Distribution by year of case receipt (independent of IFN $\beta$-1a formulation). * Data for 2014 are locked at May 3. HSA human serum albumin, IFN interferon, $s c$ subcutaneous 


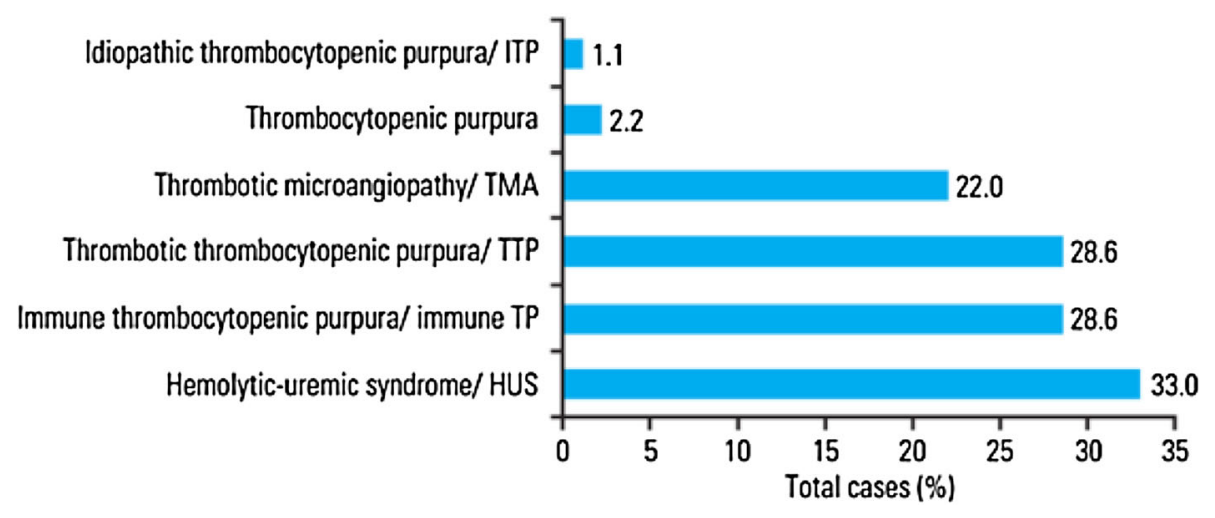

Fig. 2 Percentage of patient cases described by the search term

in the supplementary material). Early prodromal syndrome or specific patterns were not detected, although 54.9\% $(n=50 / 91)$ of patient cases had insufficient information reported about the course of the event.

Seven of $91(7.7 \%)$ patients had a fatal outcome. Five of these deaths were secondary to other causes (myocardial infarction, sepsis, hemorrhagic shock, acute respiratory distress syndrome, and septic/cardiogenic shock) and the remaining two deaths had insufficient information concerning the cause of death. Of the 84 patients without a fatal outcome, 44 of $91(48.4 \%)$ patients recovered, recovered with sequelae, or were reported as recovering; $32(35.2 \%)$ patients had not recovered at the time of the report; and the outcome was not reported or unknown in eight $(8.8 \%)$ cases. sc IFN $\beta$-1a treatment was discontinued in $84.6 \%(n=77 / 91)$ patients, maintained in $7.7 \% \quad(n=7 / 91)$, and unknown in $6.6 \%$ $(n=6 / 91)$. Treatment with sc IFN $\beta-1$ a was reintroduced in one patient without recurrence of an event.

A causal association between sc IFN $\beta-1$ a treatment and TMA/TTP-HUS was suspected in $67.0 \%(n=61 / 91)$ of cases, not suspected/not related or unlikely in $13.2 \%(n=12 / 91)$, not reported/unknown in $18.7 \%(n=17 / 91)$, and "not assessable" in one case. Risk factors and/or confounding factors were present in $45.1 \%$ ( $n=41 / 91)$ of reported cases: $24.2 \% \quad(n=22 /$ 91) prior or concomitant exposure to other treatments (e.g., chemotherapeutic agents, quinine/thiamine, and ibuprofen), $12.1 \%$ ( $n=11 / 91)$ hypertension, $4.4 \%(n=4 / 91)$ use of tobacco, $\quad 3.3 \% \quad(n=3 / 91)$ hypercholesterolemia, and $2.2 \% \quad(n=2 / 91)$ exposure to $E$. coli serotype. Other risk/confounding factors were each listed in two or fewer cases. For the remaining $54.9 \%$ of patients with no confounders reported there was generally insufficient information reported.

\section{Case Presentation: August 1, 2014}

to November 30, 2014

No increase in patient case reporting was observed in the short period (August 1, 2014November 30, 2014) following circulation of the DHCP letters. In total, six serious patient cases were reported during the time period of August 1, 2014 to November 30, 2014. Five cases were reported following the circulation of the DHCP letters from August 12, 2014: two patient cases were reported in Germany and one patient each in France, Netherlands, and Canada. 
Patient characteristics for cases reported after August 12, 2014 were similar to the May 3, 2014 data lock. Four of five patients were female. TMA was reported in three cases, and TTP and HUS were each reported in one case. No patients had a fatal outcome. Three patients recovered or were reported as recovering, one patient had not recovered at the time of the report, and the outcome was unknown in one case. sc IFN $\beta-1 \mathrm{a}$ treatment was discontinued in three patients, maintained in one patient, and reintroduced in one patient without recurrence of an event.

\section{Reporting Rates}

The estimated cumulative exposure to sc IFN $\beta-1 \mathrm{a}$ in the post-marketing setting as of May 3, 2014 was 1,259,436 patient-years. Overall reporting rate of TMA/TTP-HUS, including medically and non-medically confirmed patient cases, was 7.2 per 100,000 patient-years ( $n=91 / 1,259,436)$.

Overall, 46 patients received the HSAcontaining formulation and 35 received the HSA-free formulation (five patients received the HSA-free formulation only and 30 patients had received both formulations in countries where the HSA-free formulation was launched); 10 patients had insufficient information relating to the start date for the $\mathrm{AE}$, and/or the dates of sc IFN $\beta$-1a treatment, and were therefore assessed as 'unknown' for the formulation received at the time of the event. All patients known to have received both formulations experienced the onset of TMArelated AEs $>6$ months after the launch date of the new formulation, and were therefore all considered as HSA-free sc IFN $\beta$-1a cases.

Estimated cumulative exposures for each formulation in the post-marketing setting as of May 3, 2014 were: HSA-free formulation, 455,535 patient-years; HSA-containing formulation, 803,901 patient-years. Reporting rates for the HSA-containing and HSA-free formulations were 5.72 and 7.68 per 100,000 patient-years, respectively. The reporting rate for the five patients receiving the HSA-free sc formulation only (minus patients who received both formulations) was 1.1 per 100,000 patientyears.

\section{DISCUSSION}

Practice guidelines for TTP and TMA address the difficulties in diagnosis of the disorders due to overlap with autoimmune disease and state that drugs (including quinine, estrogen-containing medications, and pegylated IFN used to treat hepatitis C) appear to be responsible for $<15 \%$ of all TTP cases [12]. Nevertheless, no fixed guidelines exist for TMA cases associated with sc IFN $\beta-1$ a due to the rarity of the disorder and, following a recent cluster of patient reports in South Scotland [11], it was deemed that research was required to identify any differences in incidence rates associated with the introduction of a HSA-free formulation of sc IFN $\beta$ - 1 a. The present cumulative review of patient case reports from a Global Safety Database for sc IFN $\beta$-1a treatment estimated the overall reporting rate of TMA/TTP-HUS (medically and non-medically confirmed) as 7.2 per 100,000 patient-years using patientexposure data up to May 3, 2014, which further supports the rarity of TMA/TTP-HUS in this patient population. In addition, no notable association between increased frequency of TMA/TTP-HUS and the HSA-free formulation of sc IFN $\beta$ - 1 a could be detected. Reporting rates for the HSA-containing and HSA-free formulations were comparable (5.72 and 7.68 per 100,000 patient-years, respectively), and suggest that the increase in frequency of TMA/ 
TTP-HUS associated with sc IFN $\beta$ - 1 a treatment is not associated with the introduction of the HSA-free formulation.

Reporting of TMA associated with sc IFN $\beta-1 \mathrm{a}$ treatment generally increased per year over the defined search period. Although the possibility remains that this observation is related to a specific increase in TTP-HUS associated with sc IFN $\beta-1 \mathrm{a}$, we propose that it is instead related to an overall increase in reporting of TMA in general. In addition, using a separate, albeit relatively short, period of August 1, 2014 to November 30, 2014, we observed no increase in reporting rates following the circulation of DHCP letters between August 12, 2014 and October 3, 2014, which suggests that TMA/TTPHUS was already well reported prior to the safety update.

A number of patient characteristics resulting from our dataset are worthy of discussion in relation to the existing limited literature. The majority $(76.9 \%)$ of identified patient cases were female, which is consistent with both the underlying disease of MS and the limited literature for TMA associated with sc IFN $\beta-1 \mathrm{a}$ treatment $[2,13]$. Although reviews of case reports have observed cases of early onset of TMA presentation following sc IFN $\beta-1 \mathrm{a}$ treatment $[2,11]$, our dataset identified no obvious prodromal syndrome; time to onset varied from 2 months to 14 years, with a mean of 4.5 years.

The presence of patient risk factors and confounding factors should also be considered in the rare reporting of TMA/TTP-HUS associated with sc IFN $\beta$-1a treatment. Of 91 patients identified in our dataset, $45.1 \%$ presented with risk factors and/or confounding factors for TMA, including prior or concomitant exposure to chemotherapeutic agents, quinine/ thiamine, and ibuprofen; hypertension; use of tobacco; hypercholesterolemia; and exposure to
E. coli serotype. Ibuprofen is used to alleviate flu-like symptoms in patients with MS during treatment with IFN $\beta$-1a [14]. Case reports have presented ibuprofen use in patients who developed TMA [15] and TTP [16], although an association has not been reported in patients with MS who received sc IFN $\beta$ - 1 a treatment. Regarding E. coli exposure, recent cases of TMA/ TTP-HUS associated with sc IFN $\beta$ - 1 a treatment were reported in Canada [2] and Scotland [11], and were not geographically localized. In particular, Scotland is known to have higher rates of infection with E. coli O157 than other parts of the UK or Europe [17], and previous surveillance of childhood HUS in Scotland identified $E$. coli $\mathrm{O} 157$ in $>90 \%$ of cases.

This cumulative review of a Global Safety Database has some limitations. Statistical analysis of data was not feasible as the database includes a population of uncertain size. Patient-exposure data are based on market syringe sales and not patient use, while low adherence or treatment holidays are not accounted for. Consequently, calculation of patient exposure from post-marketing data tends to be underestimated. Due to the difficulty in diagnosing TMA/TTP-HUS [12], some cases may go unreported or may be misdiagnosed. Analysis of data is reliant on the reports included in the safety database and may be affected by differences between countries in reporting of AEs. Despite these limitations, the majority of TMA/TTP-HUS cases associated with sc IFN $\beta$-1a within the literature are reported as case reports and this review represents the first detailed analysis of the rare condition in patients with MS.

Subcutaneous IFN $\beta-1 \mathrm{a}$ has a wellcharacterized safety profile and 20 years of follow-up data [18-22], and the excellent benefit-risk balance of sc IFN $\beta-1$ a remains positive. Results from this study alone do not 
suggest the need for additional risk mitigation measures for different sc IFN $\beta$-1a formulations. As no fixed guidelines exist for the diagnosis and treatment of IFN $\beta$-1a-associated TMA, this potential rare condition may be difficult to diagnose and should be considered in MS patients treated with IFN $\beta$-1a during routine pharmacovigilance monitoring. Special warnings and precautions in the SmPC include monitoring for worsening angina, congestive heart failure, or arrhythmia [10]. New clinical presentation of hypertension or headache should be evaluated carefully, and monitoring of regular blood pressure is advised. Late-onset cases have been characterized by headache, hypertension, thrombocytopenia, and kidney dysfunction [23, 24]. Latest advice from the Medicines and Healthcare Products Regulatory Agency includes vigilance for early symptoms and prompt treatment [25], as early recognition prevents cases from developing into a severe event and death [12].

\section{CONCLUSIONS}

In this cumulative review of a Rebif Global Safety Database, reporting rates show that no new signal relating specifically to increased frequency of TMA/TTP-HUS with HSA-free sc IFN $\beta$ - 1 a was detected. Routine pharmacovigilance will allow further assessment of risk and confirmation whether additional risk mitigation measures are required. The benefit-risk balance of sc IFN $\beta-1 \mathrm{a}$ remains positive.

\section{ACKNOWLEDGMENTS}

This study was funded by Ares Trading SA, Aubonne, Switzerland, a subsidiary of Merck Serono SA, and the article processing charges and open access fee were funded by Merck
KGaA, Darmstadt, Germany. Medical writing support was provided by Shaun Foley, inScience Communications, Springer Healthcare and funded by Merck KGaA, Darmstadt, Germany. All authors meet the International Committee of Medical Journal Editors (ICMJE) criteria for authorship for this manuscript, take responsibility for the integrity of the work as a whole, and have given final approval for the version to be published.

Conflict of interest. Ali-Frédéric Ben-Amor is an employee of Ares Trading SA, Aubonne, Switzerland, a subsidiary of Merck Serono SA. Anton Trochanov is an employee of EMD Serono, Inc, Billerica, MA, USA. Tanya Z. Fischer is an employee of EMD Serono, Inc, Billerica, MA, USA.

Compliance with ethics guidelines. The analysis in this article is based on previously conducted clinical studies and post-marketing surveillance reports, and does not involve any new studies of human subjects or animals. There was no change to the medical management of patients, and no randomization of participants to different groups.

Open Access. This article is distributed under the terms of the Creative Commons Attribution Noncommercial License which permits any noncommercial use, distribution, and reproduction in any medium, provided the original author(s) and the source are credited.

\section{REFERENCES}

1. Summary Safety Review-Interferon-beta Products-Thrombotic Microangiopathy. Health Canada; 2014. http://www.hc-sc.gc.ca/dhp-mps/ medeff/reviews-examens/interferon-beta-eng.php. Accessed Jan 20, 2015. 
2. Vosoughi R, Marriott JJ. Thrombotic microangiopathy in interferon beta treated multiple sclerosis patients: review of literature and report of two new cases. Mult Scler Relat Disord. 2014;3(3):321-5.

3. Barbour T, Johnson S, Cohney S, Hughes P. Thrombotic microangiopathy and associated renal disorders. Nephrol Dial Transplant. 2012;27(7):2673-85.

4. George JN. Clinical practice. Thrombotic thrombocytopenic purpura. $\mathrm{N}$ Engl J Med. 2006;354(18):1927-35.

5. Fuge R, Bird JM, Fraser A, et al. The clinical features, risk factors and outcome of thrombotic thrombocytopenic purpura occurring after bone marrow transplantation. $\mathrm{Br} \mathrm{J}$ Haematol. 2001;113(1):58-64.

6. Majithia V, Harisdangkul V. Thrombotic thrombocytopenic purpura in systemic lupus erythematosus: a frequent and severe consequence of active disease. Rheumatology (Oxford). 2006;45(9):1170-1.

7. Medina PJ, Sipols JM, George JN. Drug-associated thrombotic thrombocytopenic purpura-hemolytic uremic syndrome. Curr Opin Hematol. 2001;8(5):286-93.

8. Sekhon SS, Roy V. Thrombocytopenia in adults: a practical approach to evaluation and management. South Med J. 2006;99(5):491-8.

9. Rebif (interferon beta-1a) for subcutaneous injection. Highlights of Prescribing Information. EMD Serono Inc.; 2014. http://emdserono.com/cmg.emdserono_ us/en/images/Rebif\%20PI_Jun2014_tcm115_19765. pdf?Version=. Accessed Jan 20, 2015.

10. Rebif solution for injection in cartridges. Summary of Product Characteristics. Merck Serono Ltd; 2014. http://www.ema.europa.eu/docs/en_GB/document library/EPAR_-_Product_Information/human/000136/ WC500048681.pdf. Accessed Jan 20, 2015.

11. Hunt D, Kavanagh D, Drummond I, et al. Thrombotic microangiopathy associated with interferon beta. N Engl J Med. 2014;370(13):1270-1.

12. Scully M, Hunt BJ, Benjamin S, et al. Guidelines on the diagnosis and management of thrombotic thrombocytopenic purpura and other thrombotic microangiopathies. $\mathrm{Br} \quad \mathrm{J}$ Haematol. 2012;158(3):323-35.

13. Larochelle C, Grand'maison F, Bernier GP, et al. Thrombotic thrombocytopenic purpura-hemolytic uremic syndrome in relapsing-remitting multiple sclerosis patients on high-dose interferon beta. Mult Scler. 2014;20(13):1783-7.

14. Reess J, Haas J, Gabriel K, Fuhlrott A, Fiola M. Both paracetamol and ibuprofen are equally effective in managing flu-like symptoms in relapsingremitting multiple sclerosis patients during interferon beta-1a (AVONEX) therapy. Mult Scler. 2002;8(1):15-8.

15. Mahe J, Meurette A, Moreau A, Vercel C, Jolliet P. Renal thrombotic microangiopathy caused by interferon beta-1a treatment for multiple sclerosis. Drug Des Devel Ther. 2013;7:723-8.

16. Oregel KZ, Ramdial J, Gluck S. Nonsteroidal antiinflammatory drug induced thrombotic thrombocytopenic purpura. Clin Med Insights Blood Disord. 2013;6:19-22.

17. Pollock K. Enhanced surveillance of haemolytic uraemic syndrome and other thrombotic microangiopathies (ENSHURE) in 2003-2004. Health Protect Scotland; 2005. http://www. documents.hps.scot.nhs.uk/ewr/pdf2005/0518.pdf. Accessed Jan 20, 2015.

18. Ebers G, PRISMS (Prevention of Relapses and Disability by Interferon $\beta-1$ a Subcutaneously in Multiple Sclerosis) Study Group. Randomised double-blind placebo-controlled study of interferon beta-1a in relapsing/remitting multiple sclerosis. Lancet. 1998;352(9139):1498-504.

19. PRISMS (Prevention of Relapses and Disability by Interferon $\beta-1$ a Subcutaneously in Multiple Sclerosis) Study Group, the University of British Columbia MS/MRI Analysis Group. PRISMS-4: longterm efficacy of interferon-beta-1a in relapsing MS. Neurology. 2001;56(12):1628-36.

20. Kappos L, Traboulsee A, Constantinescu C, et al. Long-term subcutaneous interferon beta-1a therapy in patients with relapsing-remitting MS. Neurology. 2006;67(6):944-53.

21. Kappos L, Verdun di Cantogno E, Cornelisse P, Kuhle J, Uitdehaag B. Cumulative dose-effect of up to 15 years of subcutaneous interferon beta-1a on clinical outcomes in relapsing-remitting multiple sclerosis: exploratory analysis of patients from the PRISMS study. J Neurol. 2012;259(Suppl 1):S110 [P470].

22. Schwid SR, Panitch HS. Full results of the Evidence of Interferon Dose-Response-European North American Comparative Efficacy (EVIDENCE) study: a multicenter, randomized, assessorblinded comparison of low-dose weekly versus high-dose, high-frequency interferon beta-1a for relapsing multiple sclerosis. Clin Ther. 2007;29(9):2031-48. 
23. Broughton A, Cosyns JP, Jadoul M. Thrombotic microangiopathy induced by long-term interferonbeta therapy for multiple sclerosis: a case report. Clin Nephrol. 2011;76(5):396-400.

24. Modrego PJ, Gazulla J. Arterial hypertension induced by interferon beta $1 \mathrm{~b}$ in a patient with multiple sclerosis. Mult Scler. 2012;18(11):1655-6.
25. Interferon-beta: risk of thrombotic microangiopathy and risk of nephrotic syndrome. Medicines and Healthcare Products Regulatory Agency; 2014. http://www.mhra.gov.uk/home/ groups/dsu/documents/publication/con465961. pdf. Accessed Jan 20, 2015. 